\title{
Clinical efficacy of anti-Mullerian hormone inspection in supporting diagnosis for climacteric disorders
}

\author{
Takao Namiki ${ }^{1}$, Haruka Kakikura ${ }^{2}$, Yukari Matsumoto ${ }^{2}$, Ueno Koichi ${ }^{2,3}$, Hiromi Sato ${ }^{2}$, Atsushi Chino ${ }^{1}$, \\ Akito Hisanaga $^{1}$, Akiyo Kaneko ${ }^{4}$, Toshiaki Kita ${ }^{4}$, Maki Kihara ${ }^{5}$, Makio Shozu ${ }^{5}$, Katsutoshi Terasawa ${ }^{1}$ \\ ${ }^{1}$ Department of Japanese-Oriental (Kampo) Medicine, Graduate School of Medicine, Chiba University, Chiba, Japan; \\ ${ }^{2}$ Department of Geriatric Pharmacology and Therapeutics, Graduate School of Pharmaceutical Sciences, Chiba Univ., Japan; \\ ${ }^{3}$ Center for Preventive Medical Science, Chiba University, Chiba, Japan; \\ ${ }^{4}$ Center for Environment, Health and Field Science, Chiba University, Chiba, Japan; \\ ${ }^{5}$ Department of Reproductive Medicine, Graduate School of Medicine, Graduate School of Medicine, Chiba University, Chiba, Japan. \\ Email: tnamiki@,faculty.chiba-u.jp; tanamiki@yahoo.co.jp
}

Received 2 July 2011; revised 20 September 2011; accepted 18 November 2011.

\section{ABSTRACT}

The diagnosis of climacteric disorders in gynecology is performed on the basis of symptoms rather than hormonal levels. Generally, the follicle-stimulating hormone (FSH) level increases when the $17 \beta$-estradiol (E2) level decreases in menopause. However, the problem of these hormones should be determined by strict timing of sampling, and there are individual specificities of decreases of hormone levels. We considered that anti-Mullerian hormone (AMH) can be measured at any time of the menstrual cycle and that it shows ovary functional decline earlier than FSH/E2, and we examined whether AMH would possibly become a good index for climacteric disorders. The subjects were 163 healthy females and 21 patients with climacteric disorders between 20 and 59 years old. We examined AMH, FSH and E2 at the same time. It is understood that in healthy females, AMH decreases with age and a decline in ovary function occurs at a relatively early age. Patients visiting clinics for climacteric disorders often have normal-range serum FSH/E2 levels, and it is clear that these values could not serve as indices of menopause at these inspections. Upon measurement of AMH in patients with climacteric disorders, most showed less than normal range ( $<14 \mathrm{pmol} / \mathrm{L}$ ), suggesting a decline in ovarian function. In addition, AMH was low in females with climacteric disorders compared with those without them. In conclusion, AMH was suggested as an objective index for climacteric disorders and possibly as a new diagnostic marker.

Keywords: Climacteric Syndrome; Anti-Mullerian Hormone (AMH), Kupperman Index(KI), Follicle-Stimulating Hormone (FSH), 17 $\beta$-Estradiol (E2)

\section{BACKGROUND}

The diagnosis of climacteric disorders in gynecology is based on symptoms rather than hormonal levels. Climacteric disorders are mainly diagnosed by subjective symptoms with questionnaires such as Kupperman index. Most symptoms of climacteric disorders are nonspecific, such as hot flashes, stiffness in the shoulders, headache, dizziness and tinnitus, which are necessary to distinguish from other diseases. Furthermore, the amount of female hormones is very important for the diagnosis of climacteric disorders. Generally, the follicle-stimulating hormone (FSH) level increases when the $17 \beta$-estradiol (E2) level decreases in menopause. However, these hormones must depend on strict timing of sampling, and there are individual differences in decreases of hormone levels. We often experienced the situation where serum FSH and E2 levels were normal or borderline regardless of patients having typical climacteric disorders diagnosed by their symptoms. Actually, in our study of climacteric disorders we defined menopause as FSH of $\geq 40 \mathrm{mIU} / \mathrm{mL}$, or as E2 of $\leq 20 \mathrm{pg} / \mathrm{mL}$ [1]. However, more than half of the patients already in menopause longer than half a year did not fulfill these criteria by random sampling. FSH might be lower than the standard, and E2 might be at a high normal level. Thus, it is difficult to diagnose climacteric disorders by hormonal levels. The levels of FSH, E2 or other hormones are not mentioned in the guidelines for hormone replacement therapy in Japan [2]. Because there are no diagnostic criteria for hormone levels, these may be cases diagnosed as a disease other than climacteric disorders when female sex hormones are at normal levels. Therefore, such cases might consult with cardiovascular or psychiatry departments. In those cases, often the cause of the symptoms is not found, and appropriate 
treatment might not be given. On the other hand, antiMullerian hormone (AMH), called Mullerian-inhibiting substance, is a Glico protein hormone of $140 \mathrm{kDa}$ belonging to the TGF-beta family [3]. The gene encoding AMH consists of five exons of $2.75 \mathrm{~kb}$ and is located in chromosome 19p33 [4]. In females, AMH is secreted from the mature ovarian follicle (pre-vesicular follicle and vesicular follicle) [5]. It is reported that the secretion ability of AMH in females increases with sexual maturation, and then is maintained at a comparatively adequate concentration. It then decreases with age, and after menopause, it is hardly secreted at all [6,7] Based on the above, AMH could become a marker of ovarian reserve, and it is now used as one of the ovarian reserve tests to predict ovary ability in assisted reproductive technology. Discovery of a new marker that can diagnose symptoms of climacteric disorders before elevation of the FSH level has been awaited. We focused on $\mathrm{AMH}$, an index of ovarian reserve. It is thought that the decrease in ovarian function is "a shift toward menopause", and AMH became a possible marker that already reflected menopause at an earlier stage. Some reports have suggested this possibility [8-10], but there is no report comparing this to the symptoms of climacteric disorders. Therefore, in this study, we aimed to investigate the correlation between AMH concentration and the symptoms of climacteric disorders and to verify whether AMH could be used as a complementary index of the diagnoses of menopause.

\section{PATIENTS AND METHODS}

\subsection{Study Objectives}

First, the age-related distribution of AMH was investigated in healthy Japanese females and compared with FSH/E2 and the AMH relation of their symptoms concerning menopause. Furthermore, in healthy females in their 20's to 50's and in patients consulting a physician due to symptoms of menopause, we examined the correlation of the severity of the symptoms with the AMH concentration.

\subsection{Subjects and Study Design}

The subjects were 163 healthy females and 21 patients with climacteric disorders aged 20 - 59 years. The age of average in healthy females and patients were $38.1+/-8.8$ years old and $49.0+/-3.8$ years old, respectively. In patients we randomly enrolled in the order they came to the outpatient. Those with a past history of disease related to ovary function (polycystic ovary syndrome [PCOS], ovarian cancer, ovarian cyst, etc.), with serum $\mathrm{AMH}$ at reference intervals of PCOS (44.98 pmol/L or higher), and the pregnant were excluded. We excluded physical dis- eases such as thyroid disease using blood examinations and psychiatric diseases such as depression by Self-rating Depression Scale (SDS) questionnaires.

We explained the objectives of the study, asked them to fill out a questionnaire (Kupperman index, menstrual cycle, past history), and obtained written consent from all subjects. We examined $\mathrm{AMH}, \mathrm{FSH}$, and E2 all at same time.

Statistical analysis was carried out as follows. The comparison between generations identifying dispersibility was done by Bartlett test. Multiple comparisons were done by Tukey-Kramer test in the case of equal dispersion or by Steel-Dwass test in the case of non-dispersion. Comparison of healthy subjects and patients with climacteric disorders identifying dispersibility was done by F-test, and examined using Student's t-test in the case of equal dispersion or Welch's t-test in the case of non-dispersion. Coefficient of correlation was obtained by Pearson's correlation test using PASW Statistics 18. Sensitivity and specificity, and the distribution of frequencies were calculated by using cross tabulation of PASW Statistics 18. StatLight 1997 (Yukms) was used for statisticcal analysis.

\subsection{Consideration of Ethical Aspects}

This study was approved by the ethical committees of the Graduate School of Medicine, Chiba University, the Graduate School of Pharmaceutical Sciences, Chiba University and the Chiba University Environment Field Science Center. In addition, as genetic information and personal medical information are critical to personal privacy, we managed all data very carefully with a stand-alone personal computer.

\section{RESULTS}

1) Hormonal measurement in healthy females (Table 1).

The concentration of serum AMH in healthy females decreased remarkably with increasing age, and had decreased significantly in all other age groups compared with the group in their 20's. As the generations rose, serum FSH concentration also rose, showing significant increases in the 40's and 50's compared with the 20's and 30 's. The concentration of serum E2 decreased signifycantly in the 50's compared with all other age groups. No significant difference was shown in Kupperman index except a tendency of increase in the 50's.

2) Application of AMH to the diagnosis of climacteric disorders.

Meaningful correlation $(\mathrm{R}=-0.244, \mathrm{P}=0.001)$ was found only in $\mathrm{AMH}$ in the examination of the correlation of each hormonal level and Kupperman index with the total healthy females and patients with climacteric disorders. There was no correlation with other hormones (Table 2). In 
Table 1. Characteristics of serum hormone levels and Kupperman index in females by age.

\begin{tabular}{ccccc}
\hline \multicolumn{3}{c}{ age group } \\
\hline & $20-29(\mathrm{n}=23)$ & $30-39(\mathrm{n}=67)$ & $40-49(\mathrm{n}=60)$ & $50-59(\mathrm{n}=13)$ \\
\hline AMH $(\mathrm{pmol} / \mathrm{L})$ & $28.85 \pm 6.59$ & $22.21 \pm 10.07^{\mathrm{a}}$ & $6.56 \pm 8.41^{\text {bd }}$ & $0.015 \pm 0.038^{\text {bde }}$ \\
FSH $(\mathrm{mIU} / \mathrm{mL})$ & $4.18 \pm 1.42$ & $5.12 \pm 4.43$ & $14.10 \pm 19.59^{\mathrm{ac}}$ & $54.71 \pm 20.08^{\text {bde }}$ \\
E2 $(\mathrm{pg} / \mathrm{mL})$ & $139.9 \pm 128.7$ & $124.1 \pm 139.8$ & $99.0 \pm 111.2$ & $14.5 \pm 14.9^{\text {bde }}$ \\
Kupperman index & $13.5 \pm 8.07$ & $14.6 \pm 9.27$ & $15.3 \pm 8.32$ & $17.6 \pm 6.67$ \\
\hline
\end{tabular}

mean \pm S.D. ${ }^{\mathrm{a}} \mathrm{P}<0.05$ compared with $20-29 ;{ }^{\mathrm{b}} \mathrm{P}<0.01$ compared with $20-29 ;{ }^{\mathrm{c}} \mathrm{P}<0.05$ compared with $30-39 ;{ }^{\mathrm{d}} \mathrm{P}<0.01$ compared with $30-39 ;{ }^{\mathrm{e}} \mathrm{P}<0.01$ compared with $40-49$.

Table 2. Correlation coefficient between score of Kupperman index and serum concentration of hormones in females.

\begin{tabular}{cccc}
\hline & AMH & FSH & E2 \\
\hline $\mathrm{R}$ & -0.233 & 0.066 & 0.055 \\
$\mathrm{P}$ & $0.001^{* *}$ & 0.371 & 0.450 \\
\hline
\end{tabular}

$* * P<0.01$.

addition, when we divided the Kupperman in- dex into two groups, medium level $(\geq 21)$ and low level $(\leq 20)$, and compared $\mathrm{AMH}$, the $\mathrm{AMH}$ concentration was significantly lower in the medium level group (Figure 1). The median of AMH concentration of these two groups was between 6 and $16 \mathrm{pmol} / \mathrm{L}$. The cut-off value of AMH was determined by examination of sensitivity and specificity and tested by Fisher's exact test. It was thought that $14 \mathrm{pmol} / \mathrm{L}$ had significantly high sensitivity and was an appropriate cut-off value of AMH (Table 3). Furthermore, we compared the hormone concentrations of patients with climacteric disorders and age-matched healthy females. AMH was significantly lower in the patients than in healthy females (Figure 2). FSH was the same in both, and E2 showed a higher tendency in the patient group compared to the healthy group, but individual differences were considerable with FSH and E2. Furthermore, when we compared the age matched healthy females with KI score over $20(n=20)$ and patients $(n=20)$, sensitivity of AMH for patients with climacteric syndrome was found to be high, whereas specificity was found to low (Figure 3). Two patients in the circle strongly suspect patients with other disease, for example depression.

Table 3. Sensitivity and specificity of Kupperman index cutoff by serum AMH levels.

\begin{tabular}{ccccccc}
\hline \multicolumn{7}{c}{ AMH (pmol/L) } \\
\hline & 6 & 10 & 13 & 14 & 15 & 16 \\
\hline sensitivity & 0.50 & 0.59 & 0.66 & 0.70 & 0.70 & 0.72 \\
specificity & 0.65 & 0.59 & 0.54 & 0.52 & 0.50 & 0.50 \\
P & 0.059 & 0.020 & 0.014 & 0.005 & 0.008 & 0.005 \\
\hline
\end{tabular}

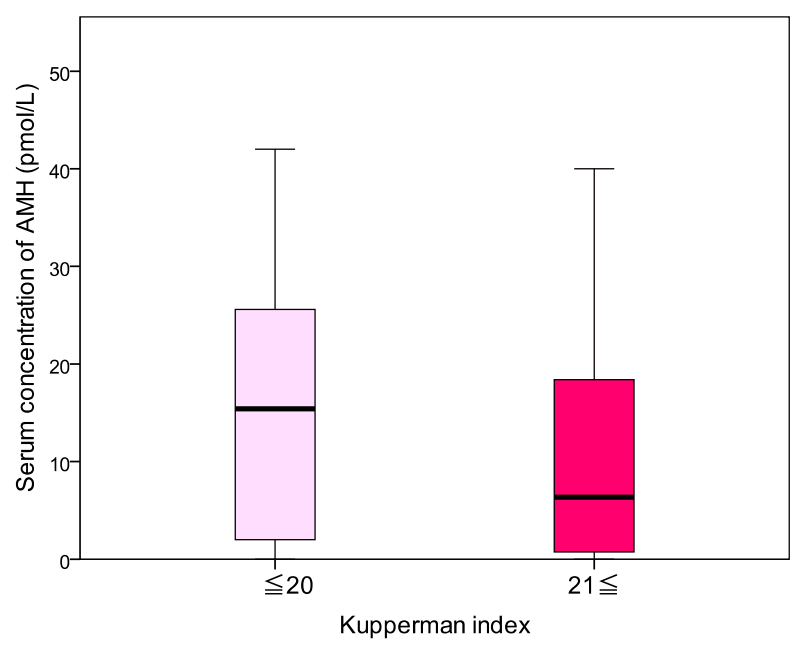

Figure 1. Comparison of Kupperman index with serum concentration of AMH: normal $(14 \leq)$ and lower $(<14)$. ${ }^{* *} P<$ 0.01 .

\section{DISCUSSION}

For the diagnosis of climacteric disorders, patients diagnosed on the basis of standard hormone values (FSH / E2) in blood samples taken at any time were very few compared with the subjective symptoms mentioned in the questionnaires. Therefore, as a candidate for a new diagnostic marker, we focused on AMH as an index of ovary function in the reproductive medicine area. As a result, a level of $\mathrm{AMH}$ under14 $\mathrm{pmol} / \mathrm{L}$ was found to be the standard concentration in most patients complaining of symptoms of climacterium (Figure 2(a)). Moreover, it is assumed that the concentration of AMH is higher in healthy females in their 40's and 50's than the sameaged patients in menopause. Then, to examine whether AMH could be used as a diagnostic marker with climacteric disorders, AMH in healthy females was measured. The results showed a remarkable decrease in every following generation (Table 1).

The change in these hormones by aging was thus confirmed in healthy females. In fact, the concentration of AMH falls under normal range in the 40's and is not even detected in most in their 50's. As for the concentrations of FSH/E2, they did not meet the criteria of meno- 
pause in both healthy females and patients with climacteric disorders, even if they were already in menopause for over 1 year (Figures 2(b)-(c)). Because we did not limit the day of measurement according to the menstrual cycle in this study, high concentrations of FSH/E2 were shown in females during the ovulation period. The concentrations of serum FSH/E2 are affected by the menstrual cycle, and it is problematic whether to use them for diagnosis of menopause or not, because the measurement day is fixed to obtain accurate data. To distinguish menopause in a normal range of hormones, a different procedure, for instance, ultrasonography, is required, and this adds to the patient's economic and time burden. Moreover, it is difficult to predict menopause from these values, as a change in FSH/E2 is not generated unless ovary function considerably deteriorates. Of

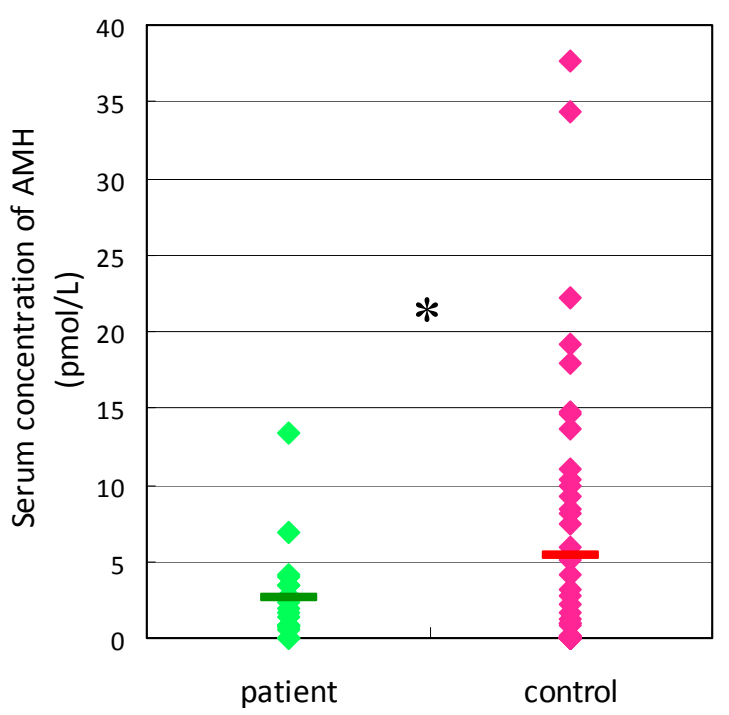

(a)

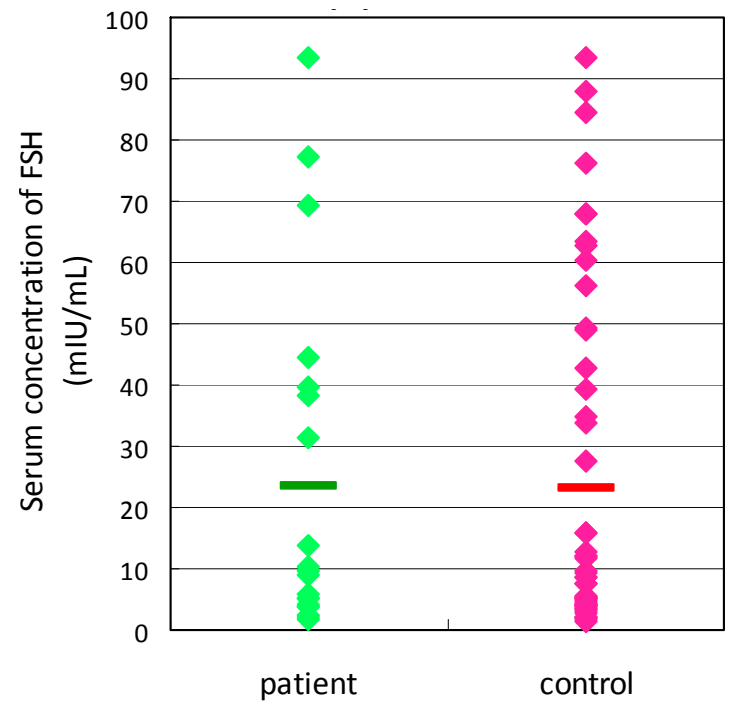

(b)

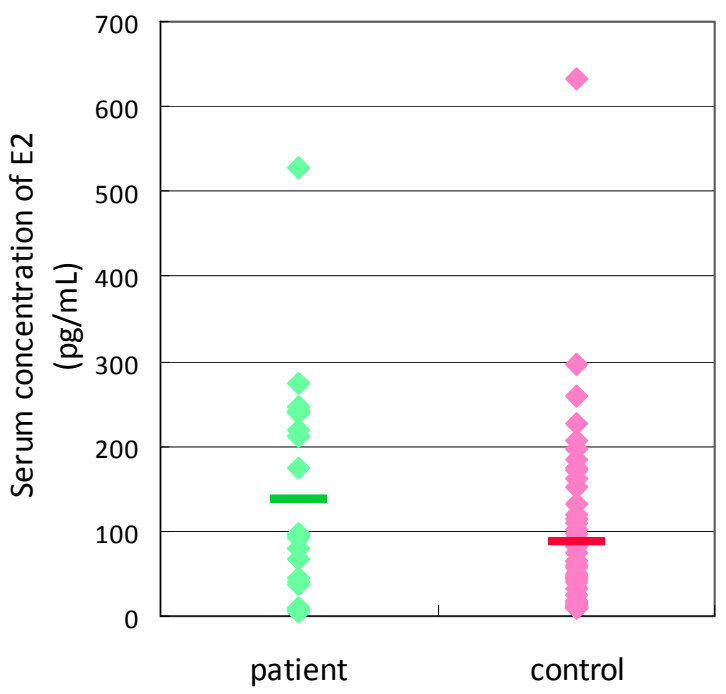

(c)

Figure 2. Comparison of serum concentrations of hormones between patients of climacteric symptoms (patients) and healthy subjects (control). $* P<0.05$.

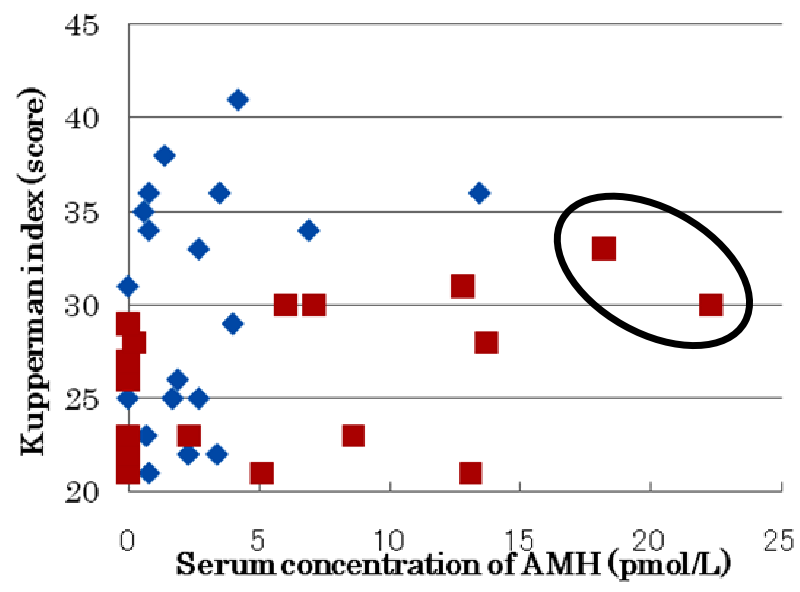

Figure 3. Relation between KI and serum concentration of AMH in females with KI score over 20 at age of 40's and 50's: control $(\boldsymbol{\square}, \mathrm{n}=20)$ and patients $(\diamond, \mathrm{n}=20)$. Two patients in the circle suspect patients with other disease or normal.

course, it is possible to use them only as an index, indicating that they are states after the completion of menopausal transition, as a definition of menopause.

On the other hand, it was reported that AMH decreases rapidly before the rise of FSH [8]. AMH was shown to remain unchanged throughout the menstrual cycle, while hormones related to female reproductive function such as $\mathrm{FSH} \cdot \mathrm{E} 2 \cdot$ luteinizing hormone $(\mathrm{LH})$ change during the menstrual cycle [8-10]. Moreover AMH does not show pulsality like FSH [11]. Thus, it is thought that AMH could possibly be a better index, as it can be measured at any time of the menstrual cycle, and it shows ovary functional decline earlier than $\mathrm{FSH} / \mathrm{E} 2$. It is actually receiving attention as a new technique of ovarian reserve 
test in the genital medicine area [12].

Next, it is generally understood that differences at the same age are relatively few, as can also be seen from the results of the present study. On this account, there is the opinion that the onset of menopause within five years can be predicted by measuring AMH [13]. In fact, AMH seems really suitable for such a prediction. The concentration of AMH was significantly low in females with strong symptoms when the level of symptoms of menopause by Kupperman index was divided into two groups; moderate degree or higher $(\geq 21)$ and light or normal $(\geq$ 20) (Figure 1). We calculated the sensitivity and specificity of these between 6 and $16 \mathrm{pmol} / \mathrm{L}$, which were the respective median values, and we searched for the cutoff value (Table 3). Sensitivity was found to be significantly high at the cut-off value of $14 \mathrm{pmol} / \mathrm{L}$. Considering that the normal range is from 14.3 to $45.8 \mathrm{pmol} / \mathrm{L}$, this was thought to be appropriate as cut-off value. In addition, AMH significantly decreased in patients with climacteric disorders when we compared AMH between such patients and normal females in their 40's and 50's. Even if comparing them at the same age, the ones with menopausal symptoms would have lower AMH, suggesting again the usefulness of AMH as an index of climacteric disorders.

The etiology of climacteric disorders has not yet become completely understood, but one cause is thought to be ovary function deterioration and resulting hormone balance collapse. AMH is secreted by follicle maturation and is concerned with control of the number of maturing follicles [14]. When menopause gets closer and the number of mature follicles decreases, AMH decreases and follicle maturation with FSH sensitivity also declines. In correspondence with this, the quantity of FSH secretion increases to cause ovulation. The decline of AMH may be one of the causes of climacteric disorders, as the balance of hormones collapses from such a process, and it is thought that various kinds of physical illnesses may occur as a result.

Activin, inhibin, GDF9, etc., which belong to the TGF$\beta$-family [15], are factors that participate in maintaining hormone balance at follicle maturation and the menstrual cycle, as well as AMH. Inhibin B (INHB) is being studied as a marker that decreases earlier than FSH in menopause $[16,17]$. INHB promotes gonadotropin-releasing hormone $(\mathrm{GnRH})$ secretion by falling before menstrualtion, and its role is thought to involve the increase of FSH secretion [18]. Taken together, an increase in the secretion of GnRH and the secretion of FSH occurs because INHB decreases at the menopausal period. Further, the secretion of INHB is greatly variable depending on the menstrual cycle. However, as INHB is still only measured at the laboratory level and, in general, its applicability is not widespread, for the present it is thought that
AMH can be readily measured, and that it can become a reliable marker compared to INHB.

In the present study, a significant correlation between $\mathrm{AMH}$ and Kupperman index was observed, and AMH fell below normal levels in most of the patients with climacteric disorders. Therefore, it was thought that $\mathrm{AMH}$ could be used as an objective marker to diagnose climacteric disorders, enabling the physician to depend not only on the patient's complaints alone. For the diagnosis of climacteric disorders, the measurement of AMH is expected to be easily carried out in the near future.

\section{CONCLUSIONS}

1) The concentration of serum AMH decreased signifycantly in healthy females with increasing age, and it was hardly detectable in their 50's.

2) Significant correlation was found between $\mathrm{AMH}$ and Kupperman index. It is clear that females with high Kupperman index and symptoms of climacteric disorders have low concentrations of AMH compared with healthy females of the same age.

\section{ACKNOWLEDGEMENTS}

This work is supported by research projects of the Ministry of Health, Labour and Welfare, Japan. The authors thank Dr. Takeshi Ouji, Hana Sugai and Ayano Ito for their help and Dr. Atsushi Yanagibori for advising us about the examinations of climacteric disorders.

\section{REFERENCES}

[1] Speroff, L. and Fritz, M. (2005) The clinical gynecologic endocrinology and infertility. 7th Edition, Lippincott Williams \& Wilkins, Philadelphia.

[2] Japan Society of Obstetrics and Gynecology \& Japan Menopause Society (2009) Guideline for Hormone Replacement Therapy 2009 (in Japanese). Tokyo.

[3] Cate, R.L., Mattaliano, R.J., Hession, C., Tizard, R., Farber, N.M., Cheung, A., Ninfa, E.G., Frey, A.Z., Gash D.J. and Chow, E.P. (1986) Isolation of the bovine and human genes for müllerian inhibiting substance and expression of the human gene in animal cells. Cell, 45, 685-698. doi:10.1016/0092-8674(86)90783-X

[4] Cohen-Haguenauer, O., Picard, J.Y., Matti, M.G., Serero, S., Nguyen, V.C., de Tand, M.F., Guerrier, D., HorsCayla, M.C., Josso, N. and Frzal, J. (1987) Mapping of the gene for anti-müllerian hormone to the short arm of human chromosome 19. Cytogenetics and Cell Genetics, 44, 2-6. doi:10.1159/000132332

[5] Vigier, B., Picard, J.Y., Tran, D., Legeai, L. and Josso, N. (1984) Another homology between sertoli and granulosa cells. Endocrinology, 114, 1315-1320. doi:10.1210/endo-114-4-1315

[6] Teixeira, J., Maheswaran, S. and Donahoe, P.K. (2001) Müllerian inhibiting substance: An instructive developmental hormone with diagnostic and possible therapeutic applications. Endocrine Reviews, 22, 657-674. doi:10.1210/er.22.5.657 
[7] De Vet, A., Laven, J.S.E., de Jong, F., Legeai, L. and Josso, N. (2002) Antimüllerian hormone serum levels: A putative marker for ovarian aging. Fertility and Sterility, 77, 357-362. doi:10.1016/S0015-0282(01)02993-4

[8] Hale, G., Zhao, X., Hughes, C., Burger, H., Robertson, D. and Fraser, I. (2007) Endocrine features of menstrual cycles in middle and late reproductive age and the menopausal transition classified according to the staging of reproductive aging workshop (STRAW) staging system. Journal of Clinical Endocrinology and Metabolism, 92, 3060-3067. doi:10.1210/jc.2007-0066

[9] Tehrani, F., Solaymani-Dodaran, M. and Azizi, F. (2009) A single test of antimullerian hormone in late reproductive-aged female is a good predictor of menopause. $\mathrm{Me}$ nopause, 16, 797-802. doi:10.1097/GME.0b013e318193e95d

[10] La Marca, A., Stabile, G., Artenisio, A.C. and Volpe, A. (2006) Serum anti-mullerian hormone throughout the human menstrual cycle. Human Repoduction, 21, 31033107. doi:10.1093/humrep/del291

[11] Sowers, M., McConnell, D., Gast, K., Zheng, H., Nan, B., McCarthy, J.D. and Randolph J.F. (2010) Anti-mullerian hormone and inhibin B variability during normal menstrual cycles. Fertility and Sterility, 94, 1432-1486. doi:10.1016/j.fertnstert.2009.07.1674

[12] Iwase, A. and Ishizuka, B. (2009) Evaluation of ovarian reserve function. (in Japanese). Acta Obstetrica et Gynaecologica Japonica, 61, 330-334.

[13] Sowers, M., Eyvazzadeh, A., McConnell, D., Yosef, M., Jannausch, M., Zhang, D., Harlow, S. and Randolph, J.
(2008) Anti-mullerian hormone and inhibin B in the definition of ovarian aging and the menopause transition. Journal of Clinical Endocrinology and Metabolism, 93, 3478-3483. doi:10.1210/jc.2008-0567

[14] La Marca, A., Sighinolfi, G., Radi, D., Argento, C., Baraldi, E., Artenisio, A.C., Stabile, G. and Volpe, A. (2010) Anti-mullerian hormone (AMH) as a predictive marker in assisted reproductive technology (ART). Human Reproduction Update, 16, 113-130.

[15] Knight, P. and Glister, C. (2006) TGF-beta superfamily members and ovarian follicle development. Reproduction, 132, 191-206. doi:10.1530/rep.1.01074

[16] Andersen, C., Rosendahl, M. and Byskov, A. (2008) Concentration of anti-müllerian hormone and inhibin-B in relation to steroids and age in follicular fluid from small antral human follicles. Journal of Clinical Endocrinology and Metabolism, 93, 2344-2349. doi:10.1210/jc.2007-2342

[17] Welt, C.K., Martin, K.A., Taylor, A.E., Lambert- Messerlian, G.M., Crowley, W.F., Smith, J.A., Schoenfeld, D.A. and Hall, J.E. (1997) Frequency modulation of follicle-stimulating hormone (FSH) during the luteal-follicular transition: Evidence for FSH control of inhibin B in normal women. Journal of Clinical Endocrinology and Metabolism, 82, 2645-2652.doi:10.1210/jc.82.8.2645

[18] Durlinger, A.L., Kramer, P., Karels, B., de Jong, F.H., Uilenbroek, J.T., Grootegoed, J.A. and Themmen, A.P. (1999) Control of primordial follicle recruitment by anti-müllerian hormone in the mouse ovary. Endocrinology, 140, 5789-5796. doi:10.1210/en.140.12.5789 